\title{
Study and development of an image synthesis software for the simulation of in-situ 3D scenes monitored by infrared cameras
}

\author{
by T. Toullier*, J. Dumoulin*, L. Mevel**
}

\author{
* Univ Gustave Eiffel, Inria, COSYS-SII, I4S Team, F-44344 Bouguenais, France \\ ** Univ Gustave Eiffel, Inria, COSYS-SII, I4S Team, F-35042 Rennes, France
}

\begin{abstract}
This study addresses the development of a software for the simulation of radiative exchanges in in-situ 3D scenes. By using the progressive radiosity method, implemented on a graphic card to improve the computation speed, a direct solution to the radiative transfer equation is proposed. The software integrates the SMARTS2 atmospheric model to take into account the solar irradiance. Moreover, a camera model has been implemented to simulate the acquisition chain from the camera's irradiance to the digital signal. To validate the software, a comparison between simulated data and actual measurements is proposed. Finally, prospects for extending the software are presented.
\end{abstract}

\section{Introduction}

Latest improvements on uncooled infrared detectors opens up new perspectives toward the low-cost thermal monitoring of civil engineering's infrastructures. Such monitoring is facing economical, security or even robustness criteria. As a consequence, sizing and placing the measurements means has become critical. In such context, a software that estimates the irradiance received by the sensors of an infrared camera has been developed. This software enables to render the radiative diffuses exchanges between the surfaces of a 3D scene, coming from a CAD (computer-aided design) for example. Thanks to the technological development of 3D animation and processing graphical units of computers, a GPGPU (general-purpose computing on graphics processing units) implementation that reduces the computation time is proposed. In order to take into account the in-situ characteristic of the scene, the software integrates the computation of the solar irradiance both diffuse and direct received at ground. Furthermore, the acquisition system has been considered by implementing a camera model that integrates the optical projection, the digitization and various noises that may affect the measurements. In this study, the developed software will be firstly introduced. Then, a validation of the algorithm for computing the radiative exchanges will be proposed. The software will then be applied to 3D static and dynamic scenes with varying parameters. Finally, prospects on hybrids methods to improve the simulation tool and consider more physical phenomenon are proposed.

\section{Progressive radiosity method for the scene irradiance simulation implemented on GPU}

The software enables the rendering of an image of a 3D scene (see Fig. 1), as perceived by an infrared camera. If different methods exist in the literature for solving such problem [2]-[4], the classical progressive radiosity method has been chosen for its physical equations solving approach. In its current implementation, the software renders the radiative exchanges between the discretized surfaces of a 3D scene. The thermal properties of the surfaces depend on the wavelength meaning that the rendering is integrated over given spectral bands. The radiosity $J_{k}$ of a surface $k$ that is given by considering its self-emission plus the contribution of the surrounding surfaces $j$ of the scene. After discretization of the equations and for a given spectral band, the following linear equations system is obtained:

$$
J_{k, \Delta \lambda_{i}}=M_{k, \Delta \lambda_{i}}+\left(1-\epsilon_{k, \Delta \lambda_{i}}\right) \sum_{j=1, j \neq k}^{j=N_{\text {elements }}} V_{k j} F_{k \rightarrow j} J_{j, \Delta \lambda_{i}}
$$

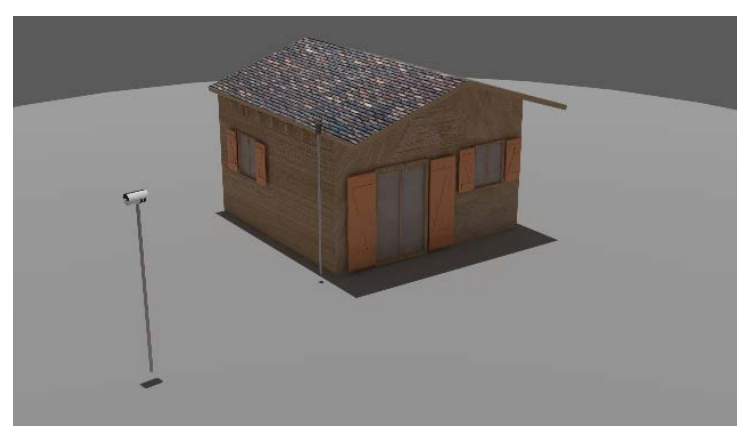

Fig. 1: 3D scene involving a wood house and an infrared camera that can be loaded by the software.

Where $V_{k j}$ represents the visibility between the element $k$ and element $j, F_{k \rightarrow j}$ is the form factor that characterizes the exchange between the surface $k$ and $j$ and $\epsilon_{k, \Delta \lambda_{i}}$ is the emissivity of the surface $k$ in the band $\Delta \lambda_{i}$. The progressive radiosity algorithm has been implemented in $\mathrm{C}++$ by taking advantage of the latest graphical libraries and parallelized through a GPGPU (General-purpose processing on graphics processing units) approach. 


\section{Infrared camera model and detectors}

Once the radiosity rendering algorithm has been implemented on accelerated hardware, a camera model has been integrated to simulate the measurements acquisition system. The camera's position, resolution, focal length, aspect ratio, etc. can therefore be customized. The rendering and visualization of the scene is made through this camera model. Furthermore, a detector model from the literature [5] has been integrated directly onto the GPU for real-time rendering and visualization. The conversion takes into account the different noises of the acquisition chain (gains, analog to digital conversion, shot noise, etc.).

\section{Solar spectral irradiance}

Under the context of civils' engineering structures monitoring, one important aspect to consider for the radiosity balance and in-situ characteristic of the measurement is the sun's contribution. Since the computation is made on a given spectral band, a spectral solar irradiance model was needed. In particular, the SMARTS2 model [1] is fully documented and provides a phenomenological approximation of the solar irradiance received at ground based on the scene's location, date and time that integrates atmospheric standard models to consider the absorption, diffusion and reflexion of the extraterrestrial radiation as it passes through the atmosphere.

\section{Applications}

Fig. 2 shows the obtained radiosity maps for a building in a static configuration. One can see that the sun radiation contribution is varying through time. Such simulation is particularly useful to evaluate the impact of the sun, the orientation of the buildings, the materials properties used on urban scenes.
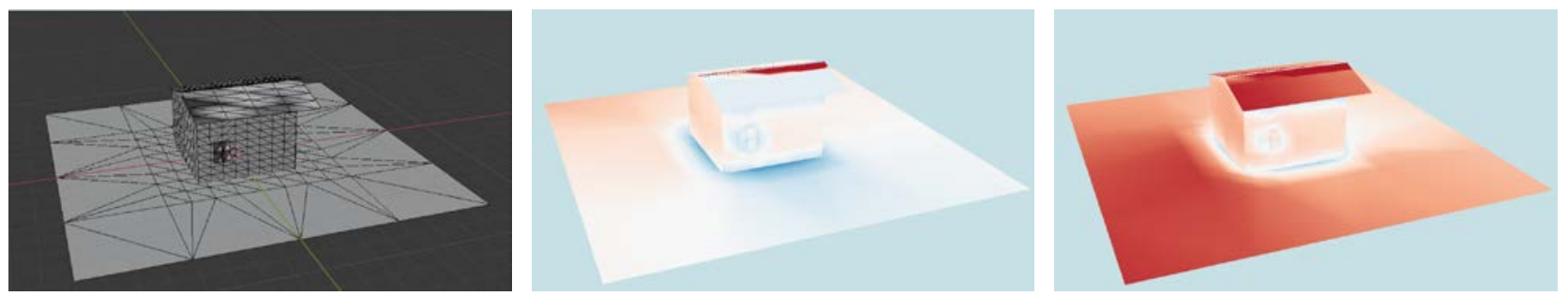

Fig. 2: Radiosity map of the wood house for a given day at different hours (from left to right: a 3D view of the model, at $6 A M$ and $12 P M)$.

\section{Conclusion}

An image synthesis software for the simulation of in-situ infrared thermography measurements 3D scene has been developed. Thanks to its implementation on recent hardware and the use of the latest rendering libraries dynamic scenes can be simulated, as long as a real-time progress rendering and the visualization of the full scene in 3D. Furthermore, the use of sensor models, camera model and atmospheric model from the literature and fully customizable enables an advanced in-situ simulation. As a consequence, advanced study can be performed prior to any exploitation with this software for sizing and placing the camera for example. However, the simulation could be improved, with for example the consideration of optical aberrations for the camera model. Moreover, one last objective will be to compare an infrared thermography measurement of a target made of different characterized materials and controlled in temperature to the simulation data.

\section{References}

[1] C. Gueymard, Simple Model for the Atmospheric Radiative Transfer of Sunshine (SMARTS2) Algorithms and Performance Assessment. 1995.

[2] P. Shirley, "Hybrid Radiosity/Monte Carlo Methods," Monte Carlo Methods, p. 24.

[3] F. X. Sillion and C. Puech, "A General Two-Pass Method Integrating Specular and Diffuse Reflection," in Proceedings of SIGGRAPH '89, 1989, pp. 335-344.

[4] J. R. Wallace, M. F. Cohen, and D. P. Greenberg, "A Two-Pass Solution to the Rendering Equation: A Synthesis of Ray Tracing and Radiosity Methods," in Proceedings of the 14th Annual Conference on Computer Graphics and Interactive Techniques, New York, NY, USA, 1987, pp. 311-320.

[5] M. Konnik and J. Welsh, "High-Level Numerical Simulations of Noise in CCD and CMOS Photosensors: Review and Tutorial," ArXiv14124031 Astro-Ph, Dec. 2014. 